This document is confidential and is proprietary to the American Chemical Society and its authors. Do not copy or disclose without written permission. If you have received this item in error, notify the sender and delete all copies.

\title{
Molecular Dynamics Simulations of Strain-Induced Phase Transition of Poly(ethylene oxide) in Water
}

\begin{tabular}{|r|l|}
\hline Journal: & The Journal of Physical Chemistry \\
\hline Manuscript ID & jp-2017-10793q.R1 \\
\hline Manuscript Type: & Article \\
\hline Complete List of Authors: & $\begin{array}{l}\text { Donets, Sergii; Institute Theory of Polymers, Leibniz-Institute of Polymer } \\
\text { Research, } \\
\text { Sommer, Jens Uwe; Leibniz-Institut für Polymerforschung, Theory of } \\
\text { Polymers }\end{array}$ \\
\hline
\end{tabular}

\section{SCHOLARONE ${ }^{\text {M }}$ \\ Manuscripts}




\title{
Molecular Dynamics Simulations of
}

\section{Strain-Induced Phase Transition of Poly(ethylene oxide) in Water}

\author{
Sergii Donets ${ }^{\dagger}$ and Jens-Uwe Sommer*, ${ }^{*} \ddagger$ \\ $\dagger$ Institute Theory of Polymers, Leibniz-Institute of Polymer Research, 01069 Dresden, \\ Germany \\ $\ddagger$ Technische Universität Dresden, Institute for Theoretical Physics, 01069 Dresden, \\ Germany \\ E-mail: sommer@ipfdd.de \\ Phone: +49 (0)351 4658 750. Fax: +49 (0)351 4658752
}

\begin{abstract}
We study dilute aqueous solutions of PEO oligomers which are subject to an elongating force dipole acting on both chain ends using atomistic molecular dynamics. By increasing the force liquid-liquid demixing can be observed at room temperature far below the lower critical solution temperature. For forces above $35 \mathrm{pN}$ fibrillar nanostructures are spontaneously formed related with a decrease in hydrogen bonding between PEO and water. Most notable is a rapid decrease of bifurcated hydrogen bonds during stretching which can also be observed for isolated single chains. The phase-segregated structures display signs of chain ordering, but a clear signature of crystalline order is not obtained during the simulation time, indicating a liquid-liquid phase transition induced by chain stretching. Our results indicate that the solvent quality of the aqueous solution of PEO depends on the conformational state of the
\end{abstract}


chains, which is most likely related with the specific hydrogen-bond induced solvation of PEO in water. The strain-induced demixing of PEO opens the possibility to obtain polymer fibers with low energy costs since crystallization starts via strain-induced demixing in the extended state only.

\section{Introduction}

Fibers made of extended and crystallized polymer chains can have extraordinary strength such as a yield stress in the order of $10 \mathrm{GPa} .{ }^{1}$ However, to obtain such materials in practice the stretching of chains has to be achieved against the interactions of the crystal-forming polymer which requires high processing energies and still leads to imperfect molecular arrangements. Metastable polymer solutions, which can undergo a disorder-to-order transition in response to stress are therefore of great interest for developing new low energy solidification techniques. The solidification with minimal energy input will drastically reduce the costs for producing polymeric materials. ${ }^{2}$ Natural silk is an example of an efficient system that produces high performance and biodegradable materials with superb mechanical properties. ${ }^{3,4}$ The material being mechanically drawn from the silk gland into the external air undergoes a liquid-to-solid phase transition. ${ }^{4}$ The structural variation of these semi-crystalline polymers is, however, very wide. For instance, the crystallites may consist of $\beta$-sheets, $\alpha$-helical coiledcoils, collagen helices etc. ${ }^{4}$ In addition, the ordered crystalline and disordered amorphous domains possess high and low hydrogen bonding densities, respectively. ${ }^{4}$ This biological complexity hampers a physical understanding of the process of fiber formation. On the other hand, an aqueous solution of poly(ethylene oxide) (PEO) is a potential candidate also capable to undergo a phase transition as a result of loss of the hydrated structure. Thus, PEO can serve as suitable model system to study strain-induced phase transition which may guide the understanding of complex biological composites such as spider silk.

PEO is one of the best studied and widely used polymer in biomedical and technological applications. ${ }^{5} \mathrm{PEO}$ is an amphiphilic, low toxic and water-soluble homopolymer with a gen- 
eral formula $\mathrm{CH}_{3}-\left[\mathrm{O}-\mathrm{CH}_{2}-\mathrm{CH}_{2}\right]_{n}-\mathrm{O}-\mathrm{CH}_{3} \cdot{ }^{6}$ In addition to the solubility in water, PEO is also soluble in organic solvents due to its unique chemical structure with both hydrophilic and hydrophobic character. Its excellent solubility in water at ambient conditions can be explained by the match in the oxygen - oxygen interdistance in the PEO and in the structure of pure water. ${ }^{7}$ In contrast, decreased water solubility of its homologues, i.e poly(methylene oxide) (PMO) and poly(propylene oxide) (PPO), can be explained by the poor match in oxygen - oxygen interdistances. ${ }^{7}$ However, the solubility of PEO decreases upon heating and its aqueous solutions undergo phase separation at a lower critical solution temperature (LCST). ${ }^{8}$ The intriguing properties of PEO in water were already considered in previous simulation studies using different force fields and degree of coarse-graining. ${ }^{9-13}$ Recently, Dahal and Dormidontova ${ }^{14,15}$ have studied the hydration, static and dynamic properties of PEO chains both in aqueous solutions and grafted to the surface using atomistic molecular dynamics simulations.

A tensile stress introduced into the amorphous material can enhance the phase separation, if the binding or coordination of water with respect to the monomers is reduced by stretching. Holland et al. ${ }^{2}$ have found indication for transient crystallization of PEO in dilute solutions under flow. In a very recent work by Semenov et al. ${ }^{16}$ the formation of stable jets from dilute PEO solutions has been explained by an increase of the intermolecular interactions between the chains due to extension-induced orientation of macromolecules. In a combined experimental and computational study, Netz et al. ${ }^{17}$ have shown that stretching of single $\mathrm{PEO}$ chains in water is related with a release of hydrogen-bonded water which can compensate the entropic forces at high strains.

In this work we will give evidence that stretching of PEO chains in water leads indeed to liquid-liquid phase separation far below the LCST. This is demonstrated by applying a force dipole to both ends of the chains which are otherwise freely movable in the solution. We argue that this observation is related to a change in the state of hydrogen bonding due to a change in the population of conformational states involving two successive oxygens 
from gauche to trans. This is in contrast to the usual paradigm of polymer physics that the polymer-solvent interaction parameter, often referred to as the $\chi$-parameter, ${ }^{18}$ does not depend on the conformational state of the chains. As a consequence stretching of PEO chains in aqueous solutions involves forces typical for flexible polymers and only in the extended state crystallization can set in within the segregated polymer phase.

\section{Simulation Details}

All simulations in this work were performed by using the molecular dynamics package LAMMPS $^{19}$ with a velocity-Verlet integrator and an integration time step of 2 fs. MD simulations were conducted either in the isothermal-isobaric (NPT) ensemble or in the isothermal-isochoric (NVT) ensemble. The temperature and pressure were kept constant using Nose-Hoover thermostat and barostat with relaxation times of 0.2 ps and 2.0 ps, respectively. The Lennard-Jones interactions were switched to zero between $8 \AA$ and $12 \AA$. The real space electrostatic interactions were cutoff at $12 \AA$, while the long-range electrostatic interactions were evaluated using particle-particle particle-mesh method. Bonds and angles in water as well as bonds involving hydrogen and any other atom were kept rigid using the SHAKE algorithm with the relative tolerance of $10^{-4}$. The revised version of the CHARMM ether force field (c35r) was used for PEO. ${ }^{20}$ Water was represented using TIP3P model. The simulated system consists of 100 PEO chains (each with total 46 ether units) and 133341 water molecules in a periodic box with following dimensions: $\mathrm{x}=\mathrm{y}=150 \AA$ and $\mathrm{z}=210 \AA$. The concentration is well below overlap of the PEO chain under the given solvent condition. Therefore, entanglement constraints should not play an important role. For the calculation of hydrogen bonds we used geometrical criteria following the classical work of Luzar and Chandler: $d(\mathrm{D}-\mathrm{A}) \leq 3.5 \AA$ and $\angle(\mathrm{D}-\mathrm{H}-\mathrm{A}) \leq 30^{\circ} .{ }^{21}$ The conclusions about our findings of the conformation-dependent hydrogen bonding remains unchanged if different cut-off criteria are used such as proposed in Ref. ${ }^{17}$ The MD results were mainly 
analyzed using VMD software. ${ }^{22}$

Comparison of the computed force-extension curve with that measured using atomic force microscopy $(\mathrm{AFM})^{17}$ for a single molecule, see Fig. S1 (a), shows a quantitative agreement with experiment and confirms that the properties of PEO in water are well described by classical MD simulations. Simulations were carried out at temperature of $\mathrm{T}=298 \mathrm{~K}$ far below the LCST of these oligomers which is located approximately at $\mathrm{T}_{L C S T}=423 \mathrm{~K}$ in our model system.

\section{Results and Discussion}

\section{Force-induced phase-separation}

Classical MD simulations were performed to study the tensile deformation and stressinduced phase transition behavior of PEO in solution at atomic level. To this end, $100 \mathrm{PEO}$ molecules were solvated by water in a rectangular box with following dimensions: $\mathrm{x}=\mathrm{y}=$ $150 \AA$ and $\mathrm{z}=210 \AA$. The larger size in $\mathrm{z}$-direction is to ensure that the fully stretched oligomer chain will not interact with itself through the periodic boundary conditions. The oligomer considered was PEO46. The system first was equilibrated at room temperature and then a constant mechanical force dipole was applied to two end points on the PEO molecules. The sum of the forces acting on both chain ends is exactly zero, therefore center of mass motion is unrestricted by the applied forces. This implies that the ends are not fixed in space and the chains can rearrange in different phases in response to the external field. In this way the distances between two end points were continuously elongating until a plateau for the averaged end-to-end distance for a given value of externally applied force was reached.

First of all, it is worth to note that at temperature of $298 \mathrm{~K}$ and without external forces the oligomer chains are fully dissolved in water with homogeneous distribution in the simulation box. Only upon heating at around $\mathrm{T}=373 \mathrm{~K}$ the PEO-water solution starts to 

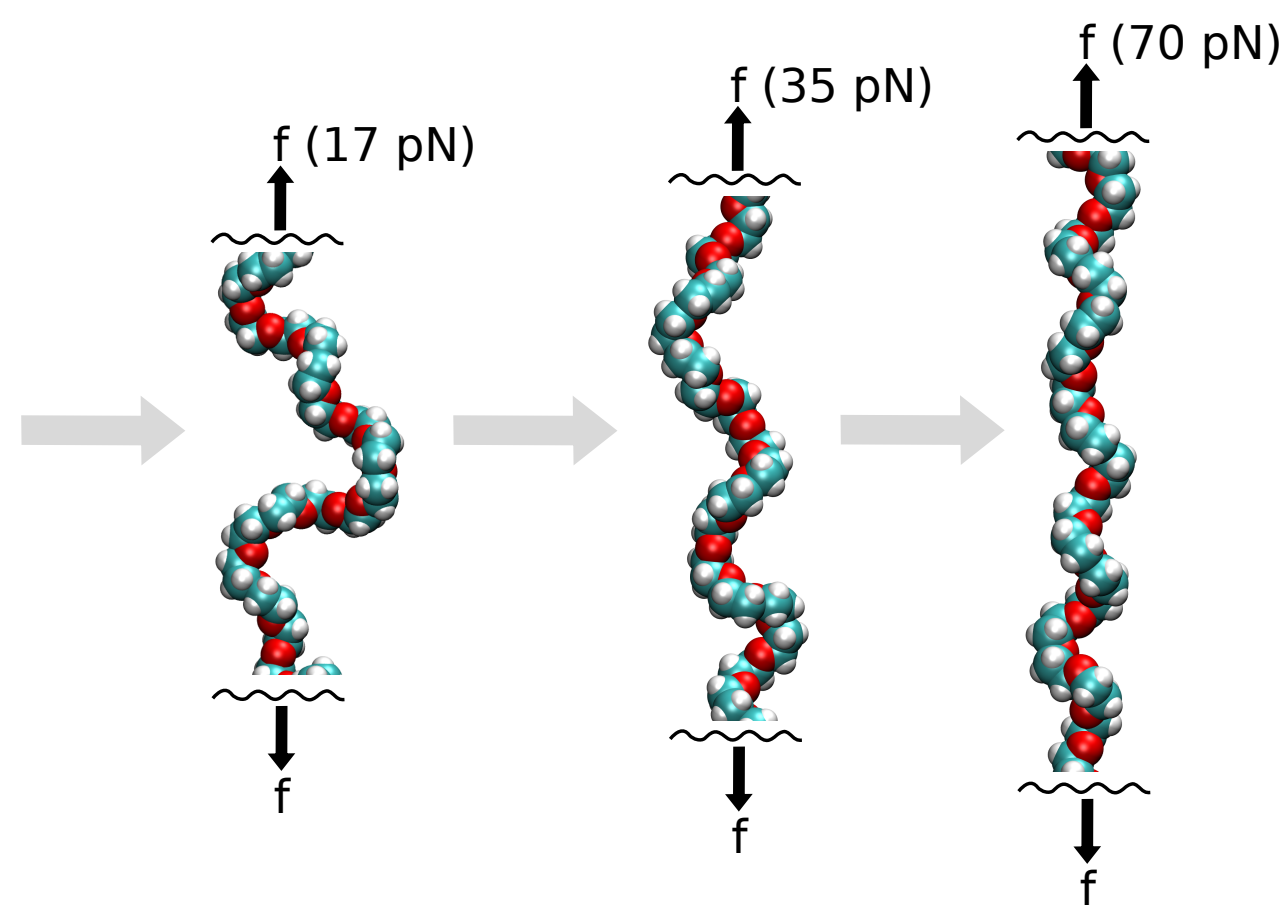

Figure 1: Snapshots of an individual PEO chain picked from the simulation box under various values of the external force. Black arrows show the directions of the applied forces. Except for the force-free chain (left) only a short sequence is displayed to illustrate the change in conformation.

phase-separate. It is known that PEO exhibits lower critical solution temperature (LCST) behavior for high enough molecular weight. The LCST of PEO with a molecular weight greater than $10^{4} \mathrm{Da}$ approaches an asymptote at temperature of $\sim 373 \mathrm{~K} .{ }^{8}$ On the other hand, according to the theoretical predictions based on the Flory-Huggins theory, which are supported by experimental measurements, the phase separation of PEO with a molecular weight of $\sim 2 \cdot 10^{3}$ Da (which was considered in this study) should occur at slightly elevated temperatures in the range of $410-440 \mathrm{~K} .^{8}$ In our own simulations using atomistic model the increase in the temperature from $\mathrm{T}=373 \mathrm{~K}$ up to $\mathrm{T}=423 \mathrm{~K}$ results in the formation of spherical aggregates of PEO molecules. The decreased solubility of PEO in water upon heating can be explained by the fact that at higher temperatures the hydrogen bond network between the ether oxygens of PEO and surrounding water molecules breaks due to thermal fluctuations, allowing the PEO-water system to demix with the formation of a two-phase region. 
During stretching the PEO conformations are altered significantly by the applied forces, as shown in Fig. 1. As we show later in more detail, deformation of the chains in response to external mechanical forces leads to the formation of highly oriented fibrillar nanostructures. However, the stress-induced phase separation can only be observed if the load is above some threshold value.

As expected, the deformation of individual chains grows monotonously with the applied force. In Fig. S1 (a) we compare the force-extension curve of the chains after relaxation with the corresponding results for single chains. Inspection of the local structure of bonds clearly proves that valence angles are not changed even up to a very high force of $350 \mathrm{pN}$ and the deformation is the result of changing the distribution of torsion angles related with a decrease of conformational entropy.

Local conformations can be classified based on the dihedral angles involving carbon and oxygen atoms along the backbone of the PEO chains. In Fig. 2 (a,c) we show probability densities for the O-C-C-O dihedral angles. We define populations of trans (gauche) conformers as the ratio between the area for the trans (gauche) conformation and the total area under the curve. The corresponding results are displayed in Fig. 2 (b,d). It is worth to note that without external forces the O-C-C-O torsion angles have a preference for the gauche conformation, which is known as the gauche effect. ${ }^{23}$ This is displayed by the black curve in Fig. 2 (a). The transformation of gauche conformers into the trans conformers requires a relaxation time which also depends on the applied force. For instance, at time of $2.0 \mathrm{~ns}$ and force of $350 \mathrm{pN}$ most of gauche conformers are transformed into trans conformers with population of about $98 \%$, see Fig. 2 (a,b). For smaller magnitudes of force, the population of trans conformers can further increase as time goes on, see Fig. 2 (c). This indicates a cooperative process between several chains which takes place at lower forces. In Fig. 2 (d) we display the two populations at the maximum relaxation time which we have applied to the systems. An abrupt change in the populations from gauche to trans occurs in the range from 70 - $140 \mathrm{pN}$, as indicated by the arrows. 

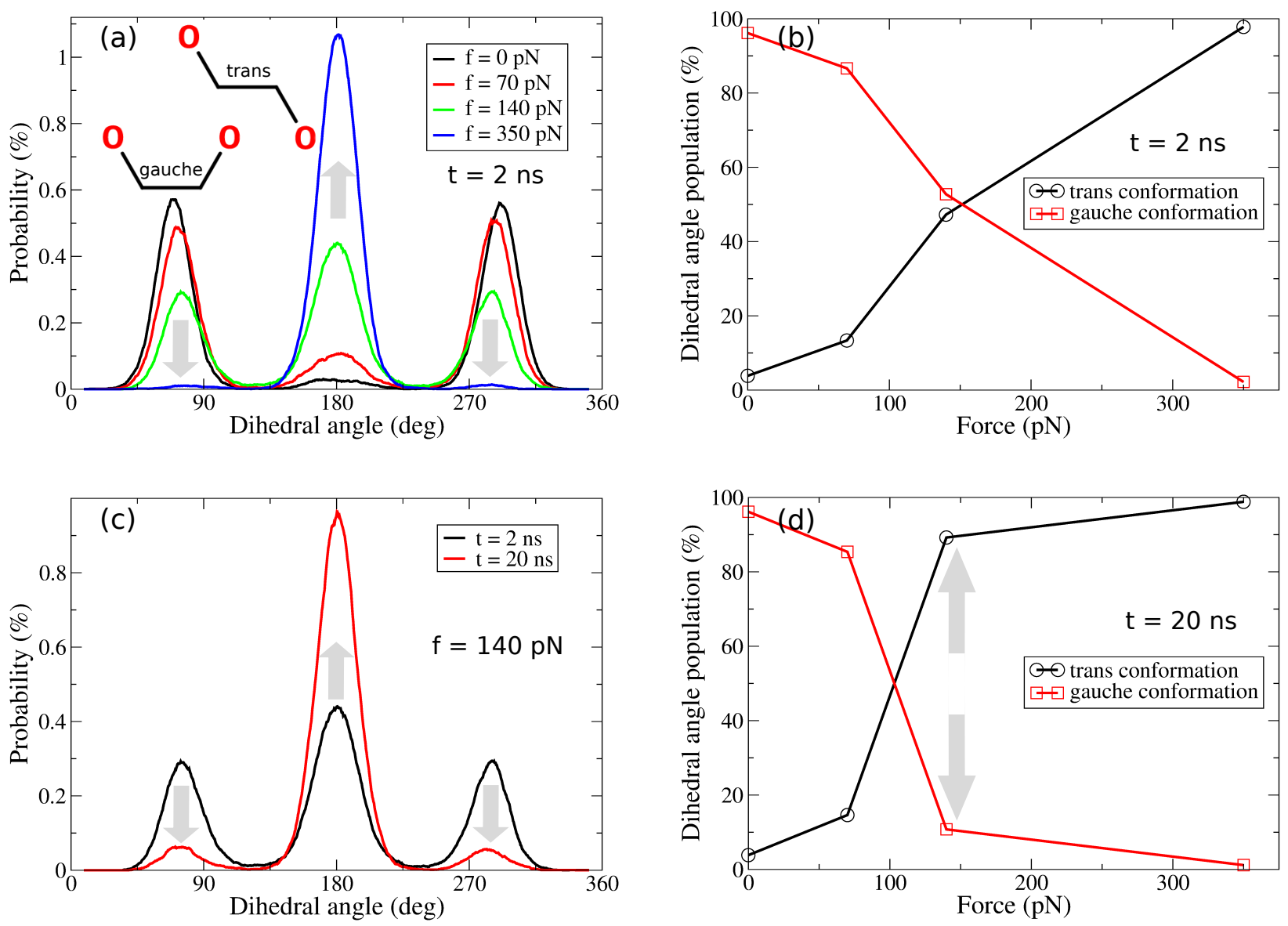

Figure 2: Normalized probability distributions of O-C-C-O digedral angles for different applied forces (a,c) and corresponding populations of conformers of PEO oligomers (b,d). The temperature is $\mathrm{T}=298 \mathrm{~K}$.

Without an external force the PEO chains are in good solvent conditions and do not aggregate. To study the impact of stretching the chains on the solubility, the two-dimensional intermolecular pair correlation functions (PCF) between the PEO monomers were calculated. The PCF is defined as:

$$
g(r)=\lim _{d r \rightarrow 0} \frac{p(r)}{2 \pi N(N / A) r d r}
$$

where $r$ is the distance between the pair of monomers, $p(r)$ denotes the average number of monomer pairs found at a distance $r$ and $r+d r$, while $N$ and $A$ are the number of reference particles and the total area of the layer perpendicular to the direction of the applied force, respectively. A two-dimensional PCF takes into account only the correlations within the layers perpendicular to the force and, as a result, this eliminates the correlations 
arising from the alignment of PEO chains along the force. These results are displayed in Fig. 3. The correlation intensities strongly depend on both the magnitude of the force
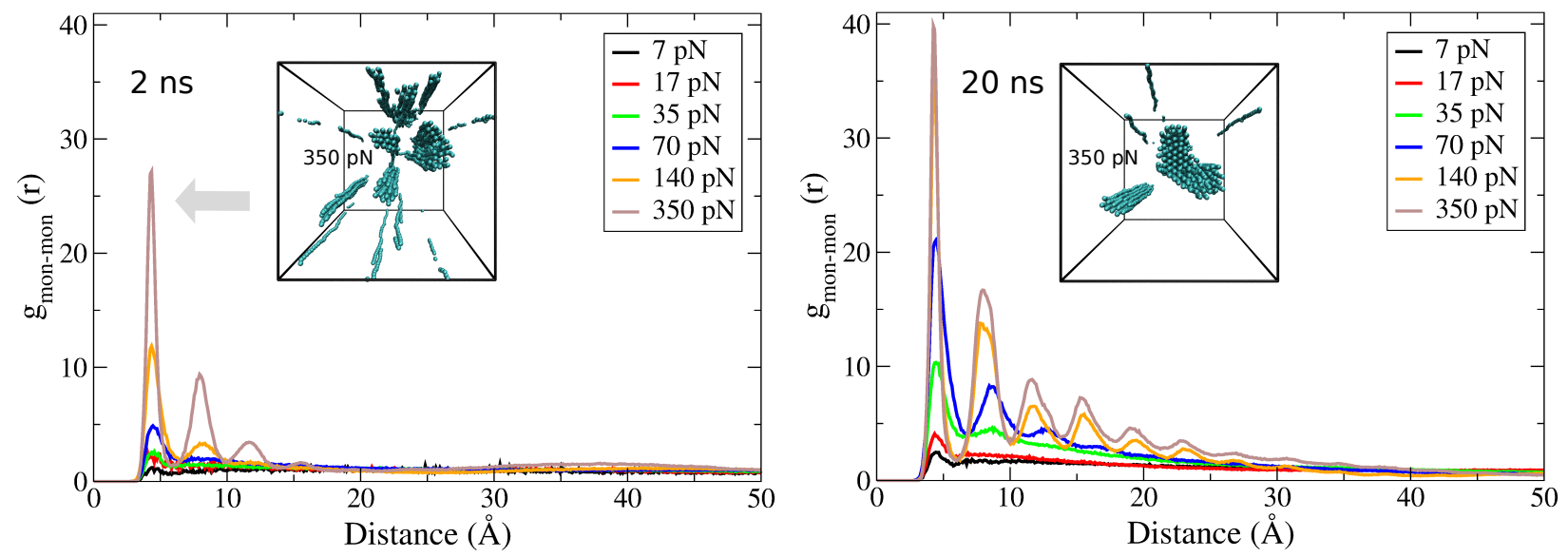

Figure 3: Two-dimensional intermolecular pair correlation function $(\mathrm{PCF})$ between the monomers of PEO oligomers at times of $2 \mathrm{~ns}$ and $20 \mathrm{~ns}$, respectively. The insets illustrate the $\mathrm{PEO}$ molecules in monomer representation (water molecules are omitted for clarity). The temperature is $\mathrm{T}=298 \mathrm{~K}$.

and elapsed simulation time (compare left versus right plots). Initially at time of $2.0 \mathrm{~ns}$ there is a big difference in correlation intensities for different forces, especially for the one of $350 \mathrm{pN}$. Nevertheless, the correlations are building-up with time and become relevant even for smaller forces, such as $35 \mathrm{pN}$ and $70 \mathrm{pN}$, as it is illustrated by the green and blue curves in Fig. 3, respectively. As the order parameter indicating the phase separation one can use the appearance of higher order peaks in the PCF. Given the relaxation time of 20 ns in our simulations, deformation-induced phase separation of PEO can be thus observed above a threshold value of the force of about $35 \mathrm{pN}$. The appearance of fibrillar clusters can be detected by an oscillating pair correlation function in Fig. 3 (right) which, however, decreases rapidly with the distance. A more detailed analysis of the scattering data, not shown here, indicates the absence of crystalline order yet. Thus, the resulting clusters should be considered as a simple demixed phase of stretched oligomers. However, it is expected that the once demixed bundles occur they are able to crystallize given a sufficiently long time for nucleation and possibly further reorganization.

To understand the origin of deformation-induced phase separation, we relate the local 
conformational changes (as shown in Fig. 2) with the change in the number of hydrogen bonds. In Fig. 4 we display our calculations of the number of intramolecular bifurcated and single hydrogen bonds, respectively. As it was mentioned above, at high enough strains the
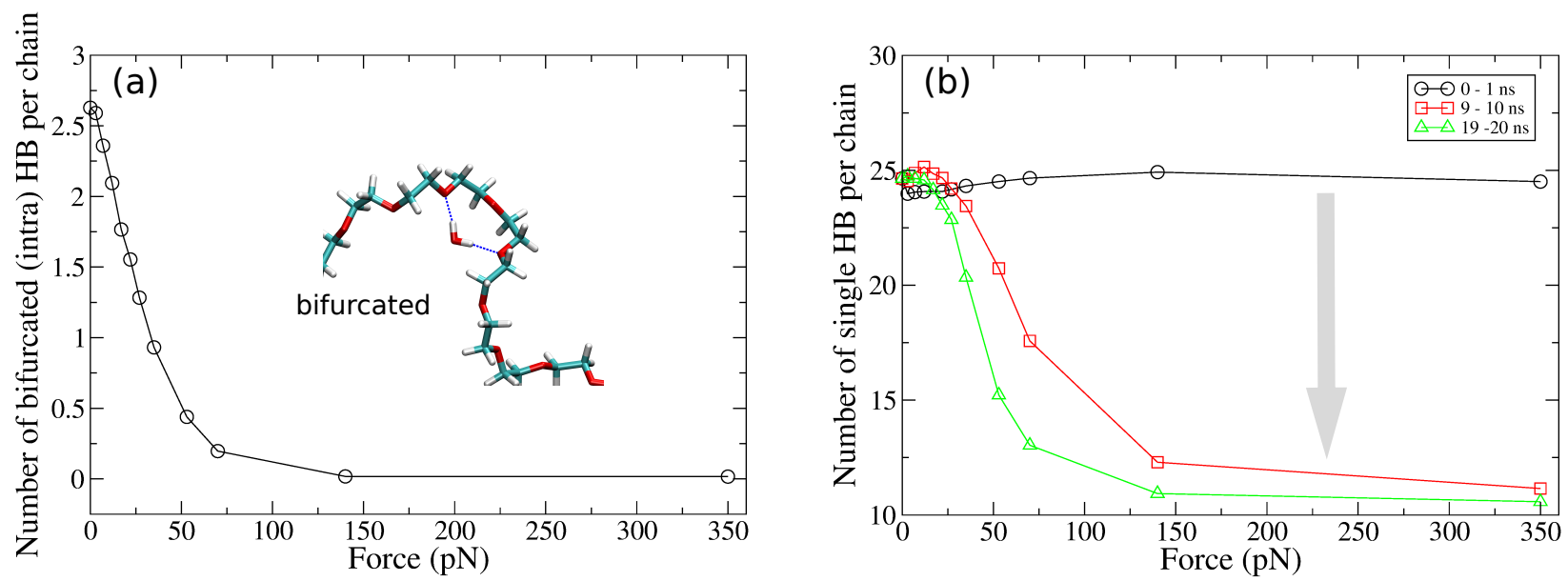

Figure 4: (a) Number of intramolecular bifurcated (obtained by averaging over last $10 \mathrm{~ns}$ of the simulation) as well as (b) single hydrogen bonds per oligomer chain as a function of force. The temperature is $\mathrm{T}=298 \mathrm{~K}$.

helical structures (enhancement of the gauche O-C-C-O and trans C-O-C-C populations) of PEO chains undergo a strong deformation, which ultimately leads to a drastic reduction in the number of intramolecular bifurcated hydrogen bonds, involving two ether oxygens within the oligomer chain and one water molecule, as shown in Fig. 4 (a). This result corresponds to that of Ref. ${ }^{17}$ These bifurcated hydrogen bonds almost completely destroyed already for the force of $70 \mathrm{pN}$. This can be explained by the steric hindrance due to the fact that hydrogen atoms of methylene bridges in trans conformation occupy certain amount of space between two adjacent oxygens, which prevents the formation of bifurcated hydrogen bonds. The number of single hydrogen bonds is displayed in Fig. 4 (b), which are also much reduced if the force reaches values of the order of $70 \mathrm{pN}$. This finding may appear somewhat counterintuitive, since the stretching of oligomers results in more extended PEO conformations in which all ether oxygens become exposed to the surrounding water molecules. However, the simulation results indicate a more than twofold reduction in the number of single hydrogen bonds from $\sim 25$ down to $\sim 11$ per one oligomer molecule for large enough 


\section{Discussion and Conclusion}

Our results clearly indicate that the stretching of oligomer chains initiates interchain aggregation, which ultimately leads to the phase separation of the PEO solution with the formation of highly oriented fibrillar nanostructures. This agrees with experimental results regarding the fiber formation, induced by shearing of PEO-water solution using SIPLI and SAXS/WAXD techniques. ${ }^{2}$ In this respect also the work by Semenov et al. ${ }^{16}$ should be noted where the authors explain their findings of the formation of stable jets from dilute PEO solutions by enhanced intermolecular interactions of elongated macromolecules. We found that the strain-induced phase transition of PEO oligomers is associated with the reduction in the number of intramolecular bifurcated as well as single hydrogen bonds. The intramolecular bifurcated hydrogen bonds are presumably responsible for the stabilization of the local helical structures. ${ }^{24}$ Accordingly, at high enough magnitudes of the force the 
helical structure elements unfold and most of gauche conformers transform into the trans conformers.

The strain-induced demixing transition occurs due to the favorable interaction energies between the PEO chains, i.e. primarily due to van der Waals interactions. In addition, the electrostatic interactions between PEO and water molecules become unfavorable as force increases, which further encourages the oligomer clustering, resulting in the loss of the hydrated structure. We note that the fibrillar structures we have obtained in our simulations are not crystalline but correspond to a concentrated polymer phase consisting of (partially) stretched chains segregated from water.

Summing up, a tensile stress introduced into the PEO-water solution reduces the solubility of the former and, if there are other oligomer chains present in the simulation box, leads to a phase separation of $\mathrm{PEO}$ from water. In terms of polymer physics this corresponds to an increase of the $\chi$-parameter due to elongation of the chains, or, in other words a conformation-depended $\chi$-parameter between PEO and water. Thus, we expect a change in the whole phase diagram of PEO in water which contains besides the temperature and concentration also the force as a coordinate. As a consequence one can expect a shift in the LCST-line in the concentration-temperature space induced by the external force. The demixed state is a possible precursor to crystallization of the extended chains and thus provides the possibility to obtain a low-energy cost stretching-crystallization pathway in the PEO solutions.

\section{Acknowledgement}

The authors thank the European Union's Horizon 2020 research and innovation programme within the project "FLow Induced Phase Transitions" (713475 - FLIPT - H2020FETOPEN-2014-2015/H2020-FETOPEN-2014-2015-RIA) for financial support and the Center for Information Services and High Performance Computing (ZIH) at TU Dresden for 
computational resources. The authors thank Olga Guskova and Oleksandr Mykhaylyk for stimulating discussions.

\section{Supporting Information Available}

Supporting information contains the results about force-extension curves of multiple PEO chains after relaxation and for single chains, as well as the results about the number of hydrogen bonds as a function of force for a single oligomer chain. In addition, intermolecular interaction energies (which include van der Waals and Coulomb energies) between PEO and PEO, and between PEO and water are also provided. This material is available free of charge via the Internet at http://pubs.acs.org/.

\section{References}

(1) OConnor, T. C.; Robbins, M. O. Chain Ends and the Ultimate Strength of Polyethylene Fibers. ACS Macro Letters 2016, 5, 263-267.

(2) Holland, C.; Vollrath, F.; Ryan, A. J.; Mykhaylyk, O. O. Silk and Synthetic Polymers: Reconciling 100 Degrees of Separation. Adv. Mat. 2012, 24, 105-109.

(3) Holland, C.; Terry, A.; Porter, D.; Vollrath, F. Natural and Unnatural Silks. Polymer 2007, 48, $3388-3392$.

(4) Walker, A. A.; Holland, C.; Sutherland, T. D. More Than One Way to Spin a Crystallite: Multiple Trajectories Through Liquid Crystallinity to Solid Silk. Proc. R. Soc. B 2015, 282, 1-9.

(5) Wang, S.; Larson, R. G. A Coarse-Grained Implicit Solvent Model for Poly(ethylene oxide), CnEm Surfactants, and Hydrophobically End-Capped Poly(ethylene oxide) and 
Its Application to Micelle Self-Assembly and Phase Behavior. Macromolecules 2015, 48, 7709-7718.

(6) Hezaveh, S.; Samanta, S.; Milano, G.; Roccatano, D. Molecular Dynamics Simulation Study of Solvent Effects on Conformation and Dynamics of Polyethylene Oxide and Polypropylene Oxide Chains in Water and in Common Organic Solvents. J. Chem. Phys. 2012, 136, 124901.

(7) Hammouda, B.; Ho, D. L.; Kline, S. Insight into Clustering in Poly(ethylene oxide) Solutions. Macromolecules 2004, 37, 6932-6937.

(8) Ashbaugh, H. S.; Paulaitis, M. E. Monomer Hydrophobicity as a Mechanism for the LCST Behavior of Poly(ethylene oxide) in Water. Ind. Eng. Chem. Res. 2006, 45, $5531-5537$.

(9) Bedrov, D.; Pekny, M.; Smith, G. D. Quantum-Chemistry-Based Force Field for 1,2Dimethoxyethane and Poly(ethylene oxide) in Aqueous Solution. J. Phys. Chem. B 1998, 102, 996-1001.

(10) Dormidontova, E. E. Role of Competitive PEOWater and WaterWater Hydrogen Bonding in Aqueous Solution PEO Behavior. Macromolecules 2002, 35, 987-1001.

(11) Dormidontova, E. E. Influence of End Groups on Phase Behavior and Properties of PEO in Aqueous Solutions. Macromolecules 2004, 37, 7747-7761.

(12) Lee, H.; de Vries, A. H.; Marrink, S.-J.; Pastor, R. W. A Coarse-Grained Model for Polyethylene Oxide and Polyethylene Glycol: Conformation and Hydrodynamics. J. Phys. Chem. B 2009, 113, 13186-13194, PMID: 19754083.

(13) Starovoytov, O. N.; Borodin, O.; Bedrov, D.; Smith, G. D. Development of a Polarizable Force Field for Molecular Dynamics Simulations of Poly (Ethylene Oxide) in Aqueous Solution. J. Chem. Theory Comput. 2011, 7, 1902-1915, PMID: 26596451. 
(14) Dahal, U. R.; Dormidontova, E. E. The Dynamics of Solvation Dictates the Conformation of Polyethylene Oxide in Aqueous, Isobutyric Acid and Binary Solutions. Phys. Chem. Chem. Phys. 2017, 19, 9823-9832.

(15) Dahal, U. R.; Wang, Z.; Dormidontova, E. E. Hydration and Mobility of Poly(ethylene oxide) Brushes. Macromolecules 2017, 50, 6722-6732.

(16) Malkin, A. Y.; Semakov, A. V.; Skvortsov, I. Y.; Zatonskikh, P.; Kulichikhin, V. G.; Subbotin, A. V.; Semenov, A. N. Spinnability of Dilute Polymer Solutions. Macromolecules 2017, 50, 8231-8244.

(17) Liese, S.; Gensler, M.; Krysiak, S.; Schwarzl, R.; Achazi, A.; Paulus, B.; Hugel, T.; Rabe, J. P.; Netz, R. R. Hydration Effects Turn a Highly Stretched Polymer from an Entropic into an Energetic Spring. ACS Nano 2017, 11, 702-712, PMID: 27977927.

(18) Gennes, P. G. d. Scaling Concepts in Polymer Physics; Cornell University Press, 1979.

(19) Plimpton, S. Fast Parallel Algorithms for Short-Range Molecular Dynamics. J. Comput. Phys. 1995, 117, 1-19, (http://lammps.sandia.gov).

(20) Lee, H.; Venable, R. M.; MacKerell, A. D.; Pastor, R. W. Molecular Dynamics Studies of Polyethylene Oxide and Polyethylene Glycol: Hydrodynamic Radius and Shape Anisotropy. Biophys. J. 2008, 95, 1590-1599.

(21) Luzar, A.; Chandler, D. Effect of Environment on Hydrogen Bond Dynamics in Liquid Water. Phys. Rev. Lett. 1996, 76, 928-931.

(22) Humphrey, W.; Dalke, A.; Schulten, K. VMD - Visual Molecular Dynamics. J. Mol. Graph. 1996, 14, 33-38, (http://www.ks.uiuc.edu/Research/vmd/).

(23) Wang, Y.-L.; Lawrence, R. S.; Lu, Z.-Y.; Laaksonen, A. Molecular Dynamics Study of Aqueous Solution of Polyethylene Oxide: Critical Test of Force Field Models. Soft Materials 2013, 11, 371-383. 
(24) Heymann, B.; Grubmüller, H. Elastic Properties of Poly(ethylene-glycol) Studied by Molecular Dynamics Stretching Simulations. Chem. Phys. Lett. 1999, 307, 425 - 432.

\section{TOC graphic}
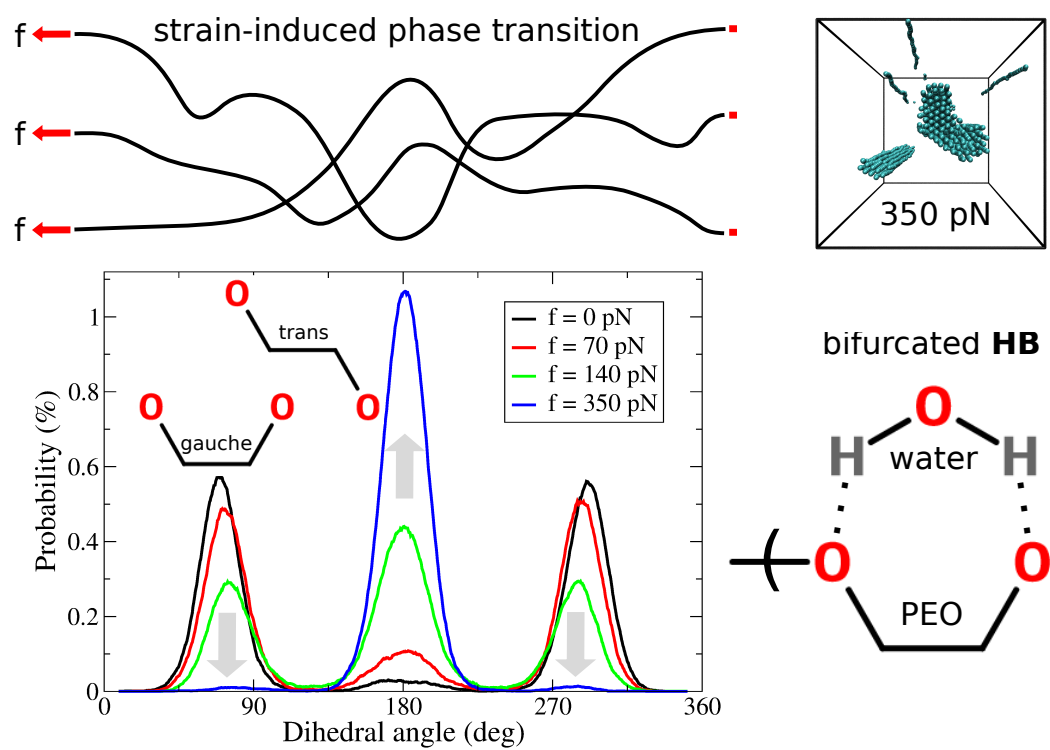

bifurcated HB

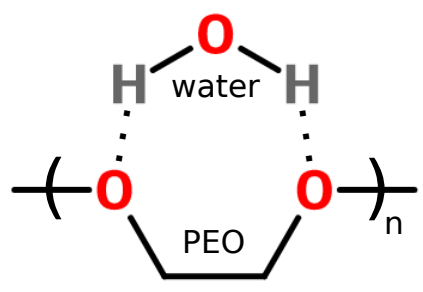


Snapshots of an individual PEO chain picked from the simulation box under various values of the external force. Black arrows show the directions of the applied forces. Except for the force-free chain (left) only a short sequence is displayed to illustrate the change in conformation.

\section{$177 \times 96 \mathrm{~mm}(300 \times 300$ DPI $)$}



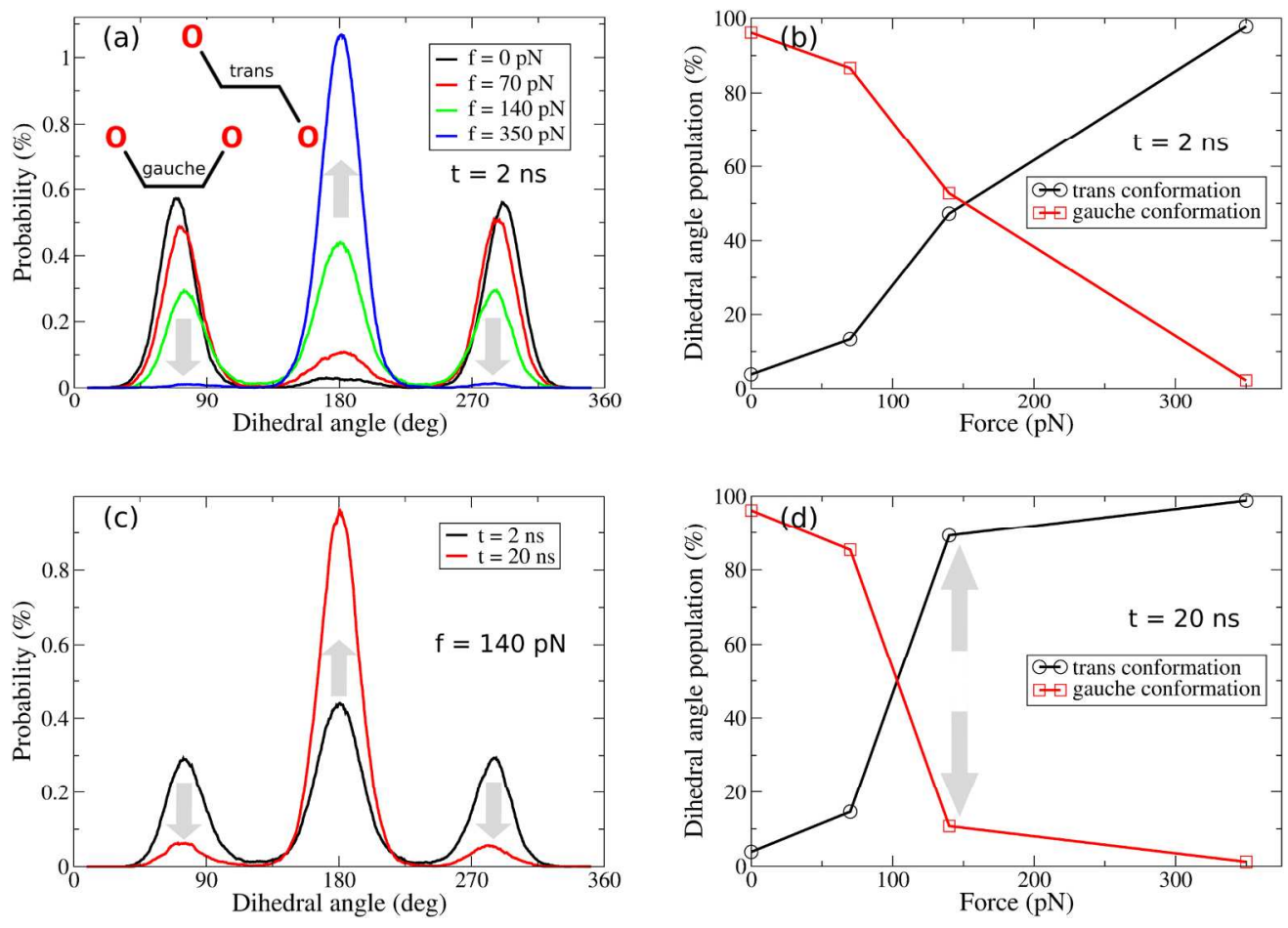

Normalized probability distributions of O-C-C-O digedral angles for different applied forces $(\mathrm{a}, \mathrm{C})$ and corresponding populations of conformers of PEO oligomers $(b, d)$. The temperature is $T=298 \mathrm{~K}$.

$177 \times 127 \mathrm{~mm}(300 \times 300 \mathrm{DPI})$ 

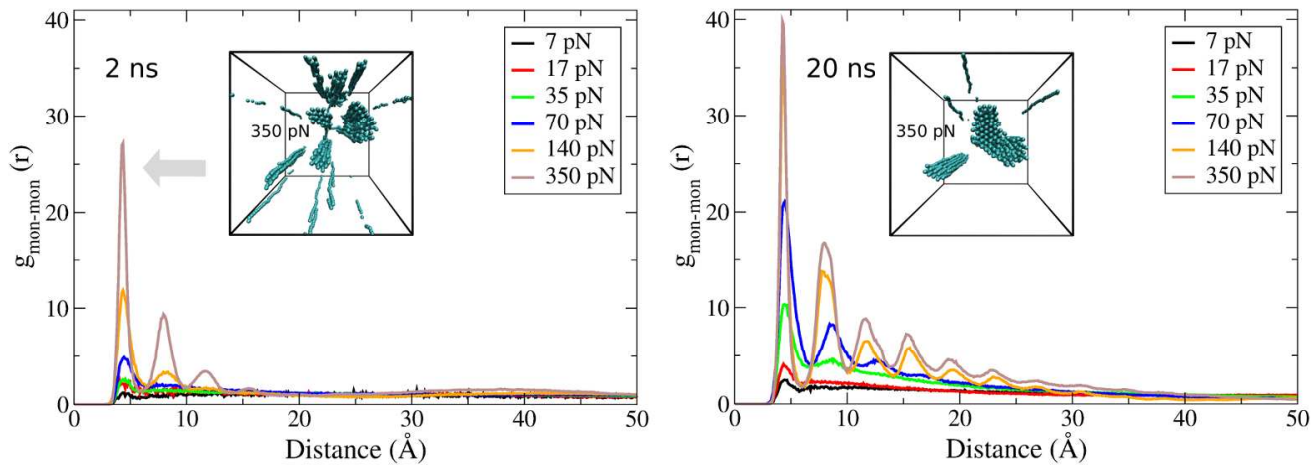

Two-dimensional intermolecular pair correlation function (PCF) between the monomers of PEO oligomers at times of 2 ns and 20 ns, respectively. The insets illustrate the PEO molecules in monomer representation (water molecules are omitted for clarity). The temperature is $\mathrm{T}=298 \mathrm{~K}$.

$177 \times 62 \mathrm{~mm}(300 \times 300$ DPI $)$ 

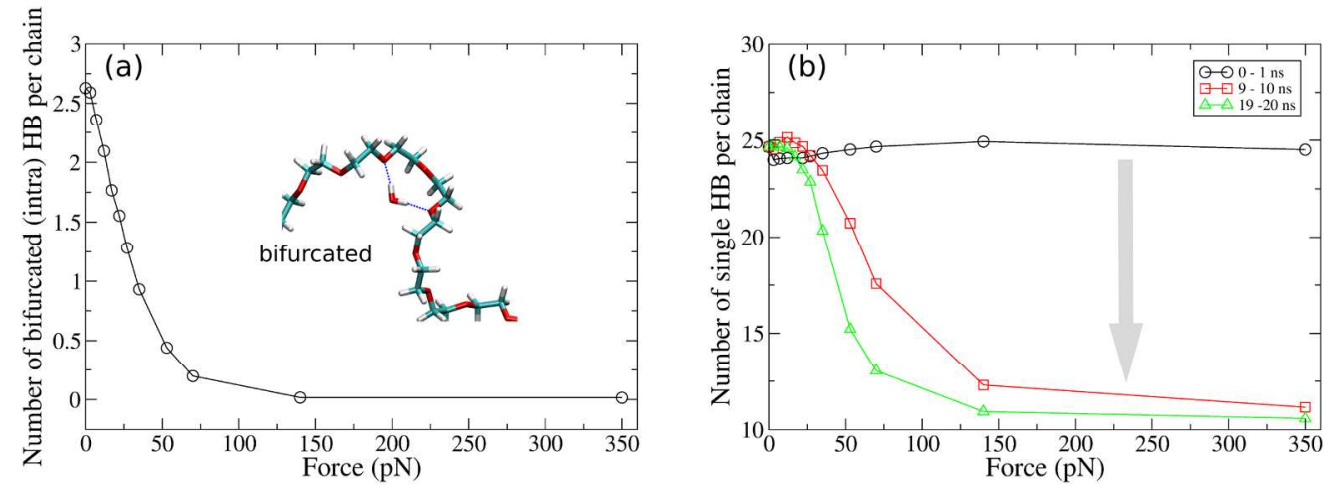

(a) Number of intramolecular bifurcated (obtained by averaging over last $10 \mathrm{~ns}$ of the simulation) as well as (b) single hydrogen bonds per oligomer chain as a function of force. The temperature is $\mathrm{T}=298 \mathrm{~K}$.

$177 \times 65 \mathrm{~mm}(300 \times 300 \mathrm{DPI})$ 


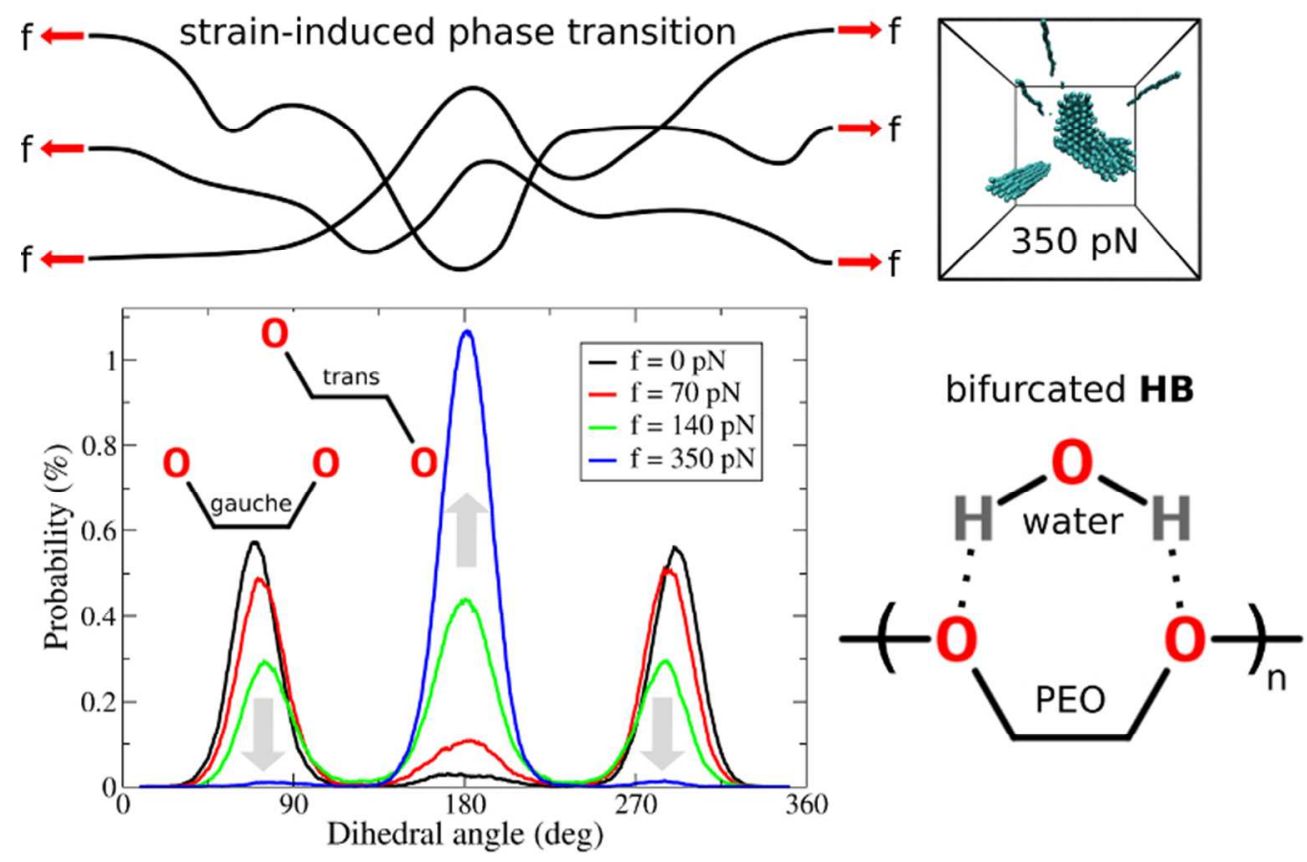

$68 \times 44 \mathrm{~mm}(300 \times 300$ DPI $)$ 